\title{
Cancer pain management - opportunities beyond the WHO ladder
}

\author{
J. Clausen \\ Universitätsklinik für Innere Medizin V, Medizinische Universität Innsbruck, Innsbruck, Austria
}

Cancer-related pain remains a significant clinical problem despite continuing advances in both antineoplastic therapy and supportive medicine. The optimal clinical management of pain can be subdivided into 3 phases:

1. Realisation of the problem by the attending medical staff, including differential classification of the individual pain syndrome, and, importantly, its quantification.

2. Choice of the most appropriate treatment modality, or a combination of multiple treatment modalities.

3. Assessment of response to the analgesic regimen, and, if required, modification of the pain management.

The first and the third of these phases will be briefly addressed in this editorial, while three short reviews in this issue of memo will focus on particular treatment modalities, which, in addition to the basic analgesic regimen, should always be considered for patients with cancer pain.

Undertreatment of cancer-related pain is not a subjective issue of a few, unlucky individuals, but is a frequent and well-documented problem, as indicated by a growing number of publications. A systematic review by Van den Beuken-van Everdingen and co-workers [1], covering literature of the past 4 decades, revealed an average cancer pain prevalence of $53 \%$ with a range from $33 \%$ in patients on curative treatment up to $64 \%$ in terminally ill patients with advanced, metastatic disease. Importantly, nearly half of the latter cohort suffers from moderate or severe pain. Stratification of the included studies by cancer type did not reveal huge differences, with head-andneck cancers leading the table with a pain incidence of $70 \%$.

A widely applied measure of the adequacy of pain management is the so-called pain management index (PMI) as proposed by Zelan, Ward and Cleeland [2, 3]. The PMI is defined as the numerical difference between the intensity of pain treatment (rated 0 to 3 , according to the WHO scale) and the original pain intensity according to the Brief Pain Inventory, categorised as 0 (no pain), 1 (1-3, mild pain), 2 (4-7, moderate pain), or 3 (8-10, severe pain). A PMI of zero or higher indicates adequate - or overtreatment, while a negative PMI defines undertreatment. Deandrea and colleagues

Correspondence: Johannes Clausen, MD, Universitätsklinik für Innere Medizin V, Medizinische Universität Innsbruck, A-6020 Innsbruck, Austria.

E-mail: johannes.clausen@i-med.ac.at recently conducted a systematic review of 26 studies including between 39 and 905 patients, respectively, published between 1994 and 2007, which assessed the PMI in cancer patients [4]. They report an average incidence of $43 \%$ for pain undertreatment with a wide inter-study range from 8 to $82 \%$. The most frequently reported factors associated with undertreatment were early disease stage (no distant metastases), a good performance score, and discrepancy between the patient's and the physician's estimate of pain severity. Furthermore, undertreatment was reported more frequently in studies from Europe or Asia than in those from the USA, in studies from poorer countries, and in studies published before 2001. Undertreatment was significantly less frequently reported from institutions specialised in cancer treatment.

More recently, the results of a telephone survey on pain in adult cancer patients, conducted in 11 European countries and Israel, was published [5], see also www.epicsurvey.com. Of 5084 patients, $56 \%$ reported to experience moderate-tosevere pain at least monthly. Of those, $23 \%$ reported to receive no pain treatment. Pain management was most frequently implemented by the responsible oncologist (42\%) or general practitioner (19\%), but rarely by a pain specialist (3\%). Of 573 patients randomly selected for a second survey phase, $77 \%$ were receiving prescription-only analgesics, with $41 \%$ taking strong opioids. Despite treatment, $63 \%$ experienced breakthrough pain. Sixty-nine percent reported pain-related difficulties with daily activities. Importantly, $50 \%$ believed that quality of life was not considered a priority by their health care professional, 33\% reported that they were not given enough time to discuss their pain, and $26 \%$ challenged their doctors' skills in managing their pain.

Taken together, this study and the above meta-analyses suggest that there is still room for improvement in cancer pain management with particular regard to communication of the problem. Additionally, an optimal management may be hampered by the physician's and/or the patient's hesitant attitude towards strong opioid analgesics, and by the inadequately frequent failure to timely adopt a multidisciplinary approach.

Facing the latter problem, the current issue of memo is dedicated to selected treatment modalities for cancer pain that may be added to the basic application of analgesics and co-analgesics. Acknowledging the role for other modalities within the field of auxiliary pain management, including physical approaches and the role of psycho-oncology, the fol- 
lowing review articles were selected to cover three basically different therapeutic areas, i.e., radiotherapy, bisphosphonates and cannabinoids.

Dr. Sztankay [6] outlines the role of radiation oncology in cancer pain management with a particular focus on bone metastases. The issue of dose fractionation is addressed from various aspects, and mechanisms underlying the well-established, various palliative effects of radiotherapy are elucidated. The article clearly illustrates the indispensable role of radiotherapy in palliation of cancer pain.

Another modality that is suited to treat or prevent pain associated with cancer affecting bone by metastatic spread or by primary involvement, is described by Dr. Haselbauer and Dr. Fiegl. Their brief review [7] elucidates the role of bisphosphonates in the treatment of cancer-related bone pain, an issue that was merely designated as secondary endpoint in most of the respective studies. Nevertheless, bisphosphonates have been shown to be effective in palliating cancerrelated bone pain besides their overall influence on skeletal-related events. In addition to the detailed and clear listing of the relevant literature, the authors provide original data from a small prospective, open-label study on the efficacy of high-dose ibandronate for refractory cancer-related bone pain, applying a "loading dose" schedule of $6 \mathrm{mg}$ for three consecutive days.

Compared to the above two modalities, the clinical use of orally administered cannabinoids for cancer pain has been studied less thoroughly, as reviewed by Dr. Kraft and Dr. Kress [8]. The authors provide a detailed view over the biology of cannabinoids, their physiological role as endocannabinoids and their relation to their endogenous receptors, $\mathrm{CB}_{1}$ and $\mathrm{CB}_{2}$. The article furthermore reviews the literature on the role of cannabis-derived compounds in palliative oncology, emphasising that their major indications are refractory anorexia and loss of appetite, as well as chemotherapy-induced nausea and vomiting. Though also approved for cancer-related pain in Canada, one has to take into consideration that cannabinoids per se have no analgesic effects and clearly have no impact on acute pain. However, possibly due to their psychotropic properties that promote extinction of aversive memories, these substances may serve as a reasonable adjunct to the basic analgesic regimen in selected cases, particularly as add-on treatment to opioids.

Despite the fact that the present compilation of articles introduces a selection of highly specialised areas of pain treatment beyond the appropriate use of potent opioids, the possibly most important issue remains to claim the full exploitation of the "WHO ladder", i.e. the timely escalation from the first to the second step, and from the second to the third.

Conflict of interest

The authors declare that there is no conflict of interest.

\section{References}

[1] van den Beuken-van Everdingen MH, de Rijke JM, Kessels AG, et al. Prevalence of pain in patients with cancer: a systematic review of the past 40 years. Ann Oncol, 18: 1437-1449, 2007.

[2] Cleeland CS, Gonin R, Hatfield AK, et al. Pain and its treatment in outpatients with metastatic cancer. N Engl J Med, 330: 592-596, 1994.

[3] Ward SE, Carlson-Dakes K, Hughes SH, et al. The impact on quality of life of patient-related barriers to pain management. Res Nurs Health, 21: 405-413, 1998.

[4] Deandrea S, Montanari M, Moja L, Apolone G. Prevalence of undertreatment in cancer pain. A review of published literature. Ann Oncol, 19: 1985-1991, 2008.

[5] Breivik H, Cherny N, Collett B, et al. Cancer-related pain: a panEuropean survey of prevalence, treatment, and patient attitudes. Ann Oncol, 2009. (Epub ahead of print)

[6] Sztankay A. Radiation therapy for palliation of cancer-related chronic pain. memo, 2(3): 173-176, (2009).

[7] Haslbauerl F, Fiegl M. Bisphosphonates as adjuvant therapy in multimodal pain management. memo, 2(3): 161-165, (2009).

[8] Kraft B, Kress HG. Cannabinoids in pain and palliative care medicine - hope or dope? memo, 2(3): 166-172, (2009). 